\title{
Vascular Compression Syndromes in the abdomen: CT imaging finding and pathophysiological correlation
}

\author{
Authors: D. Giambelluca, D. Picone, G. Caruana, R. Cannella, G. La Tona, A. Lo Casto, \\ G. Lo Re, R. Lagalla; Department of Radiology, A.O.U.P. Paolo Giaccone, Palermo, Italy
}

Vascular compression syndromes of the abdomen are a heterogeneous group of uncommon disorders, characterized by external compression of blood vessels or visceral structures by each other, which may present with a variety of nonspecific symptoms, causing delayed or wrong diagnoses. Moreover, the predisposing anatomic abnormalities of these syndromes may remain asymptomatic and found incidentally. CT is the gold standard imaging technique for many of these syndromes for its high contrast, spatial and temporal resolution and the possibility of post-processing reconstruction methods. However, it is strongly recommended to correlate radiological findings with clinical symptoms to identify the subset of patients who will benefit from possible treatment options.

- Median Arcuate Ligament Syndrome, also known with the eponym of "Dunbar syndrome", is a rare condition characterized by external compression of celiac trunk by the median arcuate ligament, which is a fibrous arch that connects the left and right diaphragmatic crura on either side of aortic hiatus: in some patients, this ligament has a low insertion point and may compress the celiac artery, determining decreased blood flow or ischemia to abdominal organs, that is clinically manifest with postprandial epigastric pain, nausea and weight loss. Sagittal reformatted MIP image of CT angiographic study will demonstrate a focal indentation on the superior surface of the celiac trunk with hooked appearance, post-stenotic dilatation and possible development of collateral vessel between celiac branches and superior mesenteric artery.

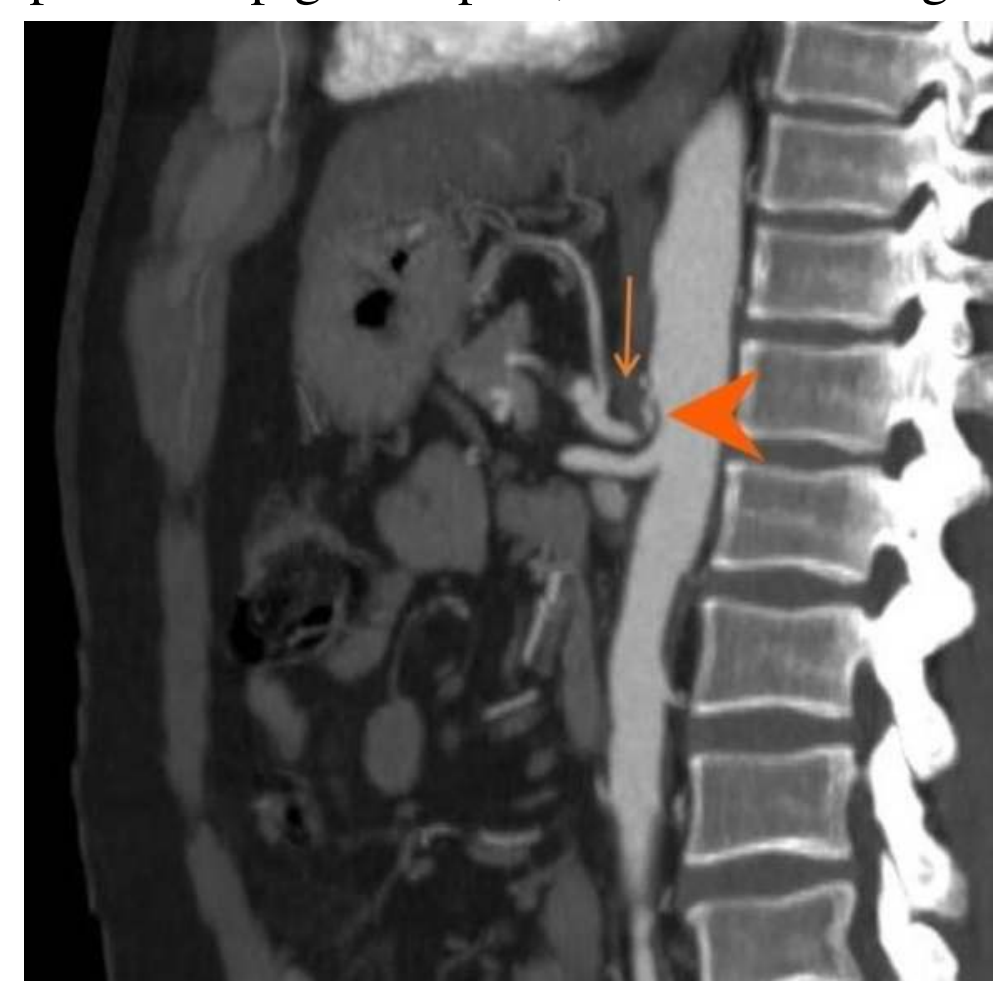

- "Nutcracker syndrome" is caused by compression of the left renal vein between aorta and superior mesenteric artery, which can lead to an increased venous pressure of the kidney, determining rupture of glomerula capillaries, with resultant intermittent hematuria. The predisposing anatomic abnormality is a narrow angle of the origin of the superior mesenteric artery from abdominal aorta to approximately $6^{\circ}-25^{\circ}$ (usually aortomesenteric angle is $45^{\circ}$, with a range between $38^{\circ}$ and $56^{\circ}$ ); however, in the absence of clinical features, this anatomic compression should be labelled as "nutcracker phenomenon".
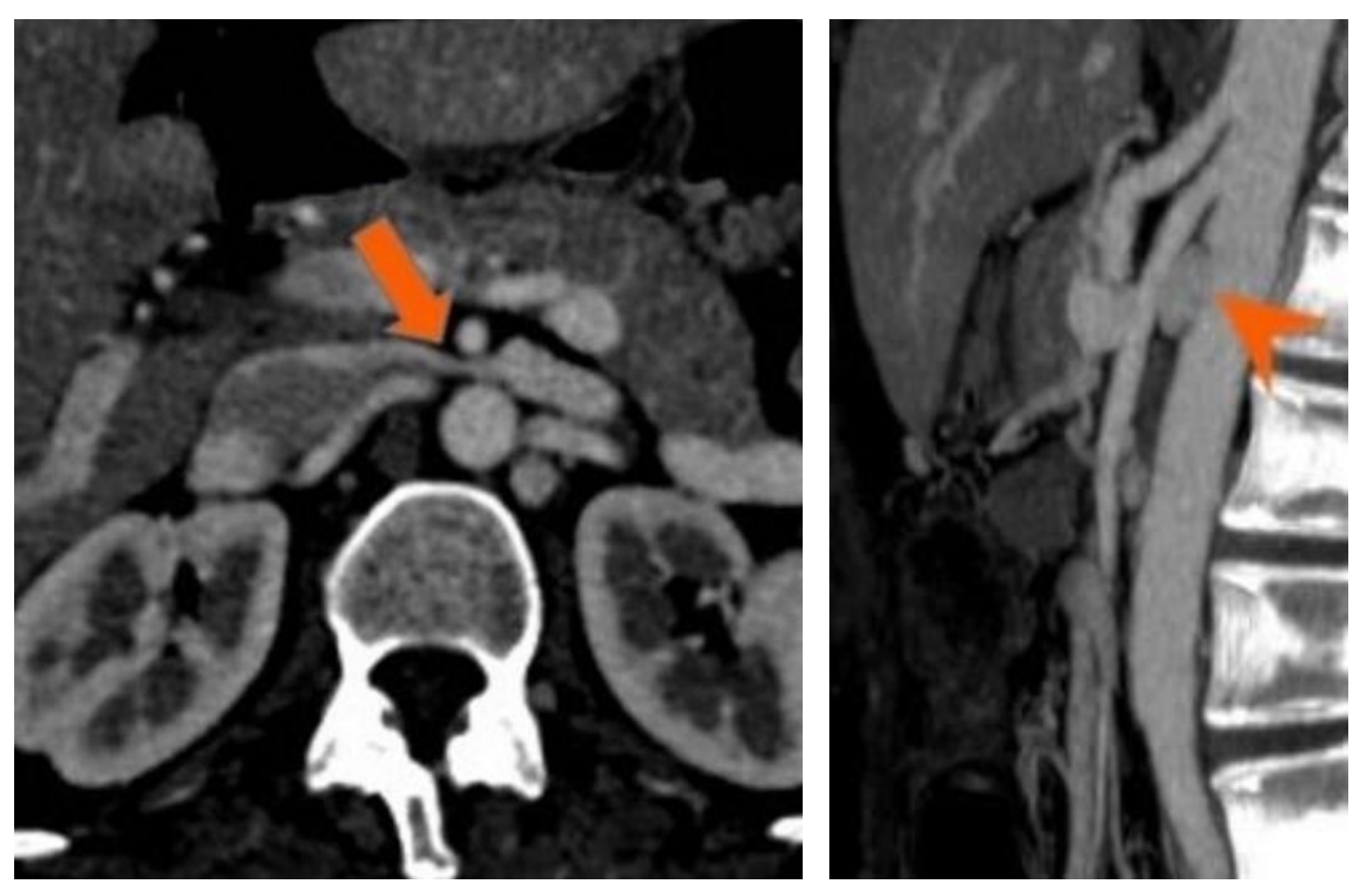

- A narrowing of the aortomesenteric angle can predispose also for "superior mesenteric artery syndrome", which is caused by vascular compression of the third part of the duodenum, associated with dilation of stomach and proximal duodenum, resulting in bowel obstruction, which clinically manifests as postprandial abdominal pain, weight loss, nausea and vomiting.

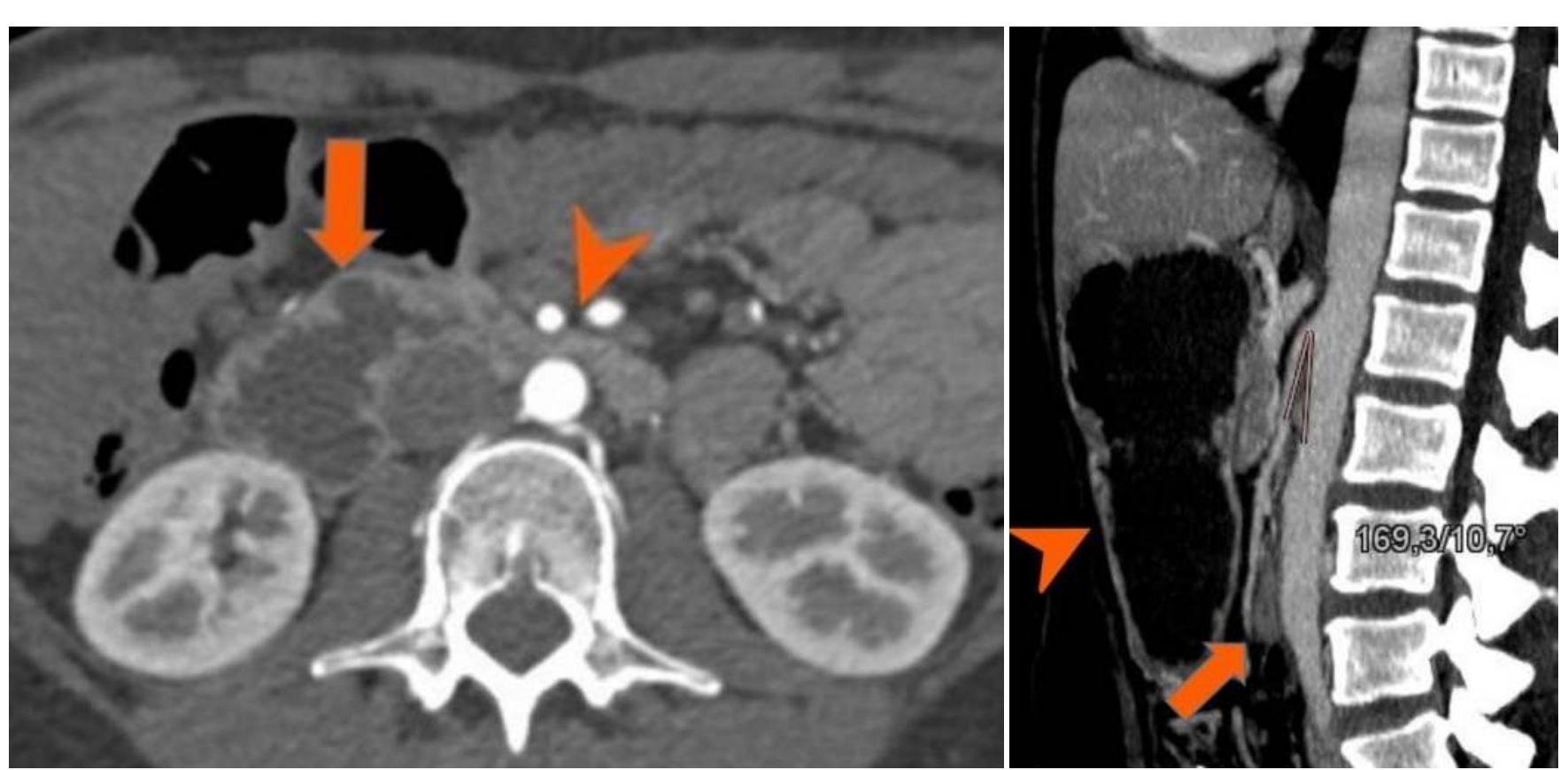

-May-Thurner syndrome" refers to a chronic compression of the left common iliac vein between the overlying right common iliac artery and the lumbar vertebrae. This condition can lead to chronic venous stasis, determining deep venous thrombosis of the left iliac and femoral veins. Post contrast CT scan allows visualization of venous compression and assessment of iliofemoral venous thrombosis extension.

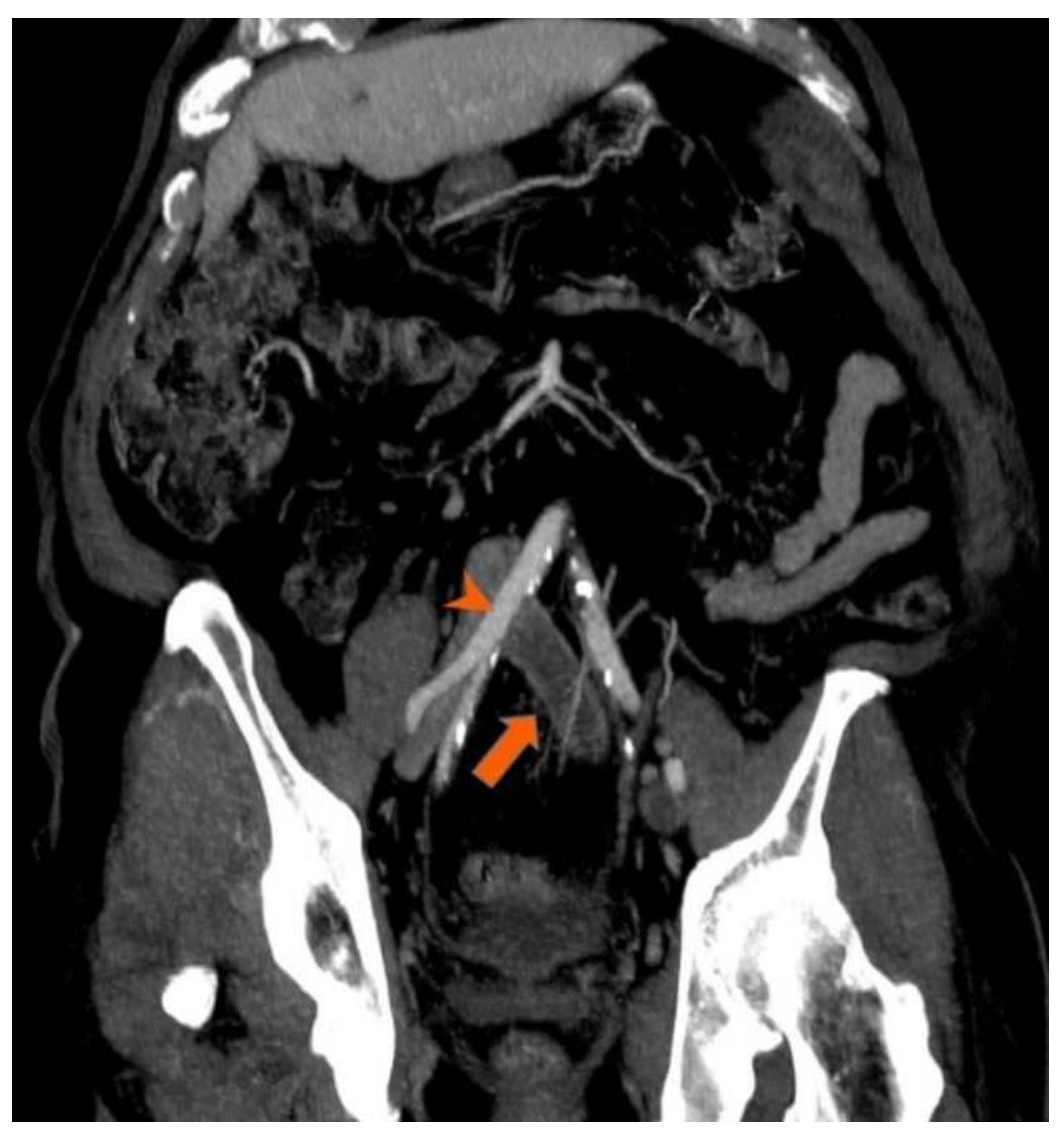

- Ureteropelvic junction obstruction (UPJO) can be caused by "crossing vessels", which pass above the ureteric transition point: in particular, it was given special attentions to lower pole segmental renal artery, which may be branch of main renal artery, or may arise separately as an accessory branch from the aorta or iliac artery. Obstructive uropathy may also occur at the point of intersection of the ureter with retroperitoneal vascular structures, usually a dilated or aberrant ovarian vein, resulting in ovarian vein syndrome (OVS). Both in the cases of UPJO than of OVS, CT scan performed in a late arterial phase allows visualization of ureteric compression by crossing vessels, with associated various degrees of hydronephrosis and proximal hydroureter.
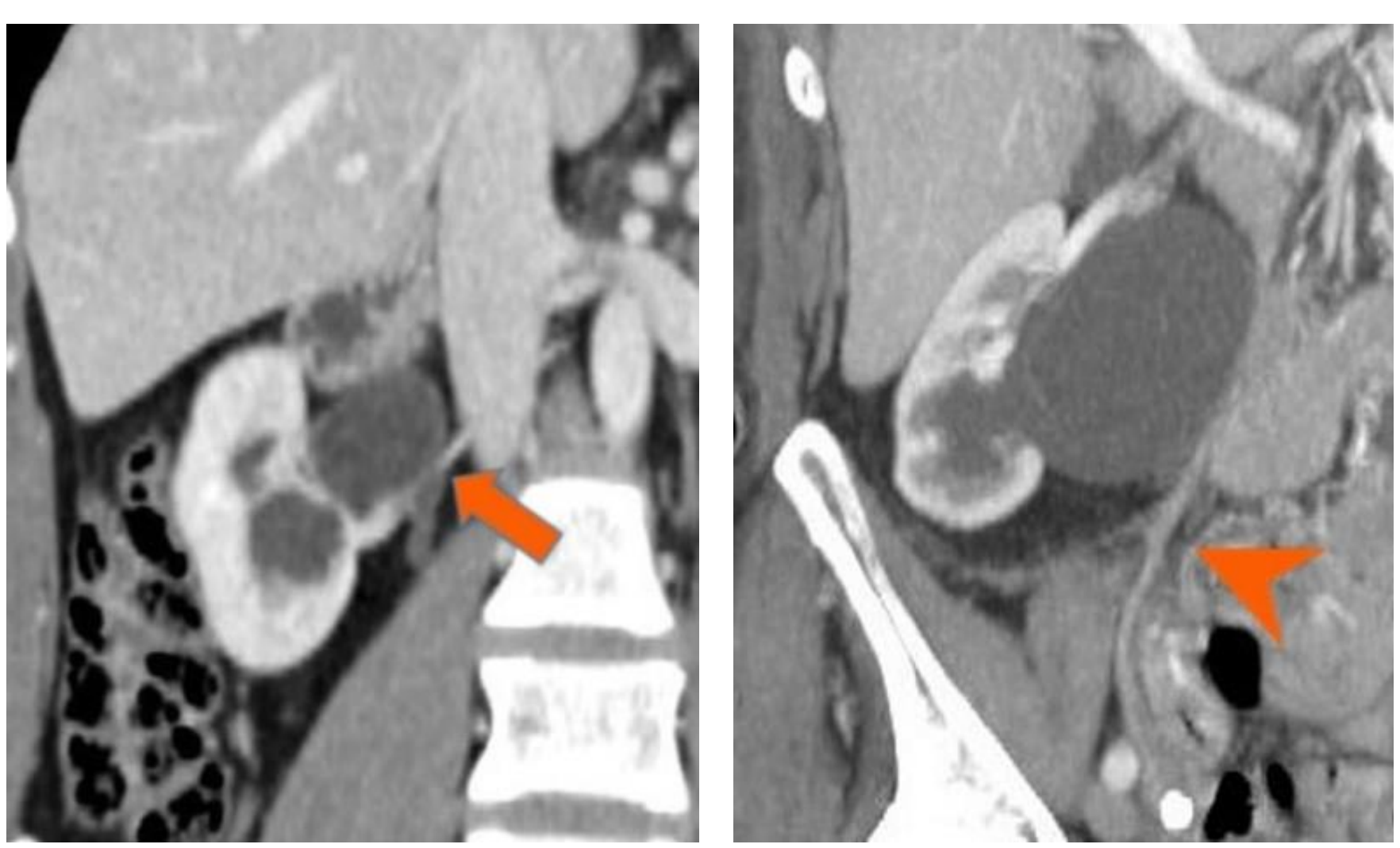